http://dx.doi.org/10.5007/1677-2954.2014v13n1p74

\title{
JUSTIÇA, LIBERDADES BÁSICAS E AS BASES SOCIAIS DO AUTORRESPEITO
}

\section{JUSTICE, BASIC LIBERTIES AND SOCIAL BASES OF SELF- RESPECT}

\author{
DENÍLSON LUÍS WERLE ${ }^{1}$
}

(UFSC, Brasil)

\begin{abstract}
Unlike those communitarians authors who interpret Rawls's theory of justice as an expression of an individualistic conception of autonomy and that would have as a central concern to guarantee individual's negative freedom, the purpose of this article is to show that the conception of social justice of Rawls is based on a plural conception of liberty ("the basic liberties") which seeks to assure to all citizens the institutional, material and intersubjective conditions for self-realization and mutual autonomy in social cooperation. The article argues that this interpretation of Rawls opens new possibilities for thinking a critical theory of justice concerned with the vulnerabilities related to the autonomy of the citizens as moral persons.
\end{abstract}

Keywords: Basic liberties. Social bases of self-respect. Theory of justice.

\section{RESUMO}

Contrário àqueles autores comunitaristas que interpretam a teoria da justiça de Rawls como expressão de uma concepção individualista de autonomia e que teria como preocupação central assegurar a liberdade negativa das pessoas, o objetivo do artigo é mostrar que a concepção de justiça social de Rawls está fundamentada em uma concepção plural de liberdade (as liberdades básicas) que procura assegurar a todos os cidadãos as condições institucionais, materiais e intersubjetivas para a autorrealização e autonomia mútuas na cooperação social. $\mathrm{O}$ artigo defende que essa interpretação de Rawls abre novas possibilidades para pensar uma teoria crítica da justiça preocupada com as vulnerabilidades relacionadas à autonomia das pessoas.

Palavras-chave: Liberdades básicas. Bases sociais do autorrespeito. Teoria da justiça.

Tornou-se um lugar comum dizer que o fundamento normativo do liberalismo consiste em assegurar a autonomia dos indivíduos e que, consequentemente, a tarefa de uma teoria da justiça seria a de fazer com que a estrutura básica da sociedade estivesse arranjada de modo a reduzir as diferentes vulnerabilidades e arbitrariedades que impedem a realização da autonomia a um mínimo social aceitável (já que seria uma tarefa impossível eliminá-las totalmente). Para muitos críticos comunitaristas, o liberalismo não

ethic@ - Florianópolis v.13, n.1, p. 74 -90, Jun. 2014. 
está a altura de sua missão principal por não ter uma teoria mais refinada das múltiplas ameaças à autonomia, e acaba, com isso, subestimando as vulnerabilidades as quais estão sujeitos os indivíduos em sua vida social mais ampla. A tese típica é que o liberalismo defende uma concepção por demais individualista de autonomia e de liberdade pessoal, fundamentada em uma idealização enganosa dos indivíduos como sujeitos autossuficientes e autoconfiantes, não levando em conta os diversos contextos sociais, econômicos e políticos de socialização e de formação dos indivíduos, os quais determinam, em grande parte, o caráter e a capacidade para a autonomia. Ele não consegue, por isso, interpretar adequadamente as exigências da justiça social, não considerando as carências, as vulnerabilidades e a interdependência social dos indivíduos. Para sanar este problema, seria necessário ampliar as exigências da justiça social segundo uma concepção ampliada de autonomia, que inclusive nos leve para além das circunstâncias institucionais e materiais da autonomia, considerando que ela é uma capacidade que existe somente no contexto das relações sociais que a asseguram e somente em conjunção com o sentido interno do que significa ser autônomo (HONNETH e ANDERSON, 2005, p.126). A ideia central dessa concepção ampliada de autonomia é a de que as pessoas, para agirem de forma autônoma, têm de ser capazes de manter certas atitudes positivas em relação a si mesmas (de autoconfiança, autorrespeito e autoestima), cuja formação depende de processos sociais de interação e de relações interpessoais de reconhecimento bem sucedidas.

Esse tipo de deficiência, apontado por diversos teóricos comunitaristas e autoras feministas, atinge uma parte significativa dos teóricos liberais. Porém, não parece fazer jus à concepção de justiça elaborada por Rawls, que, como apontam Honneth e Anderson (2005, p. 142), tem "recursos impressionantes" para lidar com as dimensões intersubjetivas da autonomia, precisamente na importância que o bem básico do autorrespeito assume na deliberação e justificação dos princípios de justiça: "só faz sentido que as partes incluam o bem intersubjetivo básico do autorrespeito em suas deliberações sobre a estrutura básica de uma sociedade justa, se eles já compreenderam que a concepção e a persecução de seus planos de vida dependem fundamentalmente da estima dos outros" (HONNEHT e ANDERSON, 2005, p. 142). ${ }^{2}$ No que se segue, pretendo mostrar a importância que Rawls atribui à noção intersubjetiva do autorrespeito 
que acaba sendo um dos critérios para justificar a própria concepção de liberdades básicas e sua prioridade. ${ }^{3}$ Argumentar dessa forma ajuda a compreender melhor o tipo de teoria da justiça oferecida pelo liberalismo político de Rawls. Ao contrário da visão estereotipada do liberalismo político de Rawls como uma teoria política da justiça orientada para a defesa de uma concepção de liberdade negativa e de autonomia privada dos indivíduos, o que pretendo mostrar é que considerando o objeto de uma teoria da justiça (a estrutura básica da sociedade), a concepção de liberdades básicas e a importância dada às bases sociais do autorrespeito, o liberalismo político de Rawls aponta para novos e interessantes caminhos para a elaboração de uma teoria crítica da justiça preocupada com as vulnerabilidades relacionada à autonomia das pessoas situadas em diferentes contextos intersubjetivos de socialização.

Um primeiro aspecto a ser mencionado é que diferentemente de um liberalismo mais tradicional, a teoria da justiça de Rawls articula diferentes dimensões da liberdade pessoal, sem que uma esteja subordinada a outra. Na formulação de Rawls a determinação do sistema adequado de liberdades fundamentais não pode ser pensada como tendo a função de maximizar um valor absoluto (a liberdade como tal); antes, tratase de justificar um conjunto de liberdades fundamentais necessárias para o desenvolvimento adequado das capacidades razoáveis e racionais da pessoa moral autônoma, como membro pleno de uma sociedade democrática. Isso fica mais ou menos claro na conferência VIII do Political Liberalism (1993), na qual Rawls afirma que foi levado a modificar sua explicação da liberdade a partir da crítica que lhe foi feita por Hart no artigo Rawls on Liberty and its priority (1975). É sabido que Hart apontou duas falhas graves no argumento de Rawls: $1^{\circ}$ ) que as razões pelas quais as partes na posição original adotariam as liberdades básicas e concordariam com sua prioridade não estavam suficientemente explicitadas em Uma Teoria da Justiça; $2^{\circ}$ ) que quando os princípios de justiça são aplicados nos estágios constitucional, legislativo e judicial não é fornecido um critério suficiente para saber como as liberdades básicas devem ser especificadas e ajustadas umas às outras quando são conhecidas as circunstâncias sociais da sociedade. Rawls pretende corrigir essas duas falhas tornando mais preciso o argumento a favor dos dois princípios de justiça, mostrando como as liberdades básicas e os fundamentos para sua prioridade podem ser justificados a partir da concepção de cidadãos como pessoas 
livres e iguais em conjunto com uma explicação dos bens básicos e uma certa concepção de cooperação social.

Para dar uma resposta consistente a Hart, Rawls começa reformulando os princípios de justiça e esclarecendo qual o conceito de pessoa que está na base da sua concepção de justiça. Os dois princípios passam a ter a seguinte formulação:

"a. Each person has an equal right to a fully adequate scheme of equal basic liberties which is compatible with a similar scheme of liberties for all.

b. Social and economic inequalities are to satisfy two conditions. First, they must be attached to offices and positions open to all under conditions of fair equality of opportunity; and second, they must be to the greatest benefit of the least advantaged members of society" (RAWLS, 1993, p. 291)

A alteração ocorre principalmente no primeiro princípio, onde a expressão "mais abrangente sistema total", presente na primeira formulação do princípio em Uma teoria da justiça, é substituída por "sistema plenamente adequado", e também é inserida "que seja" antes da palavra "compatível". O propósito dessa reformulação é mostrar justamente que "no priority is assigned to liberty as such, as if the exercise of something called 'liberty' has a proeminent value and is the main if not the sole end of political and social justice" (RAWLS, 1993, p. 291-292). Se assim fosse, o liberalismo político de Rawls acabaria sendo igual aos demais liberalismos éticos-políticos que defendem algum tipo de ideal moral abrangente.

O liberalismo político de Rawls não é abrangente justamente pelo modo como justifica as liberdades básicas e sua prioridade. Rawls fornece, primeiro, uma lista dessas liberdades fundamentais iguais no primeiro princípio de justiça: a liberdade de pensamento e de consciência; as liberdades políticas e a liberdade de associação, assim como as liberdades especificadas pela liberdade e integridade da pessoa; e, finalmente, os direitos e liberdades abarcados pelo império da lei, não atribuindo ou justificando nenhuma forma de hierarquização entre elas. O que importa nessa lista não é hierarquizar as liberdades - por exemplo, dizendo que as liberdades civis da autonomia privada seriam mais importantes do que as liberdades políticas da autonomia pública - mas sim pensar a 
formação e institucionalização de um sistema das liberdades, que permanece (e deve permanecer) em certa medida indeterminado quanto à combinação das várias liberdades conflitantes. Ao dizer que não atribui nenhuma prioridade à liberdade como tal Rawls argumenta que sua interpretação das liberdades fundamentais segue a tradição do pensamento democrático, cujo foco sempre esteve voltado "on achieving certain specific liberties and constitutional guarantees, as found, for example, in various bills of rights and declarations of the rights of man" (RAWLS, 1993, p. 292).

Dado que, em uma sociedade democrática plural, as várias liberdades fundamentais estão fadadas a conflitar umas com as outras, é necessário que as regras institucionais que definem essas liberdades, sejam ajustadas da melhor maneira possível pela prática política dos próprios cidadãos, de modo que se encaixem num sistema coerente de liberdades garantido igualmente a todos, sistema que deve estar aberto a revisões e adaptações conforme mudem as condições sociais necessárias para seu exercício duradouro, respeitando a esfera de aplicação de cada liberdade. ${ }^{4}$ Rawls continuará a manter a tese da prioridade da liberdade no sentido de que uma liberdade só pode ser limitada ou negada em nome de uma outra ou de outras liberdades fundamentais, e não por considerações de bem estar geral, concepções abrangentes da vida boa ou por valores perfeccionistas. Sendo que as liberdades fundamentais, quando entram em choque entre si, só podem ser limitadas recorrendo às próprias liberdades, fica claro que nenhuma delas pode ser considerada absoluta.

Para justificar a prioridade do sistema amplo de liberdades básicas frente às outras considerações práticas, Rawls recorre aos conceitos políticos e normativos de pessoa que estão na base do liberalismo político. Além de serem vistas como cidadãos livres e iguais, as pessoas são consideradas como cidadãos que possuem duas faculdades (ou capacidades) morais: a de terem um senso de justiça e a de formar, buscar e revisar uma concepção racional do bem. A lista de liberdades básicas sugerida por Rawls visa precisamente mostrar quais as condições sociais fundamentais para o desenvolvimento adequado e o exercício pleno dessas duas capacidades da pessoa durante a vida inteira, cuja realização é fundamental para o desenvolvimento de um senso de independência pessoal e de autorrespeito. 
Mas, como justificar a própria idéia de pessoa? Certamente, afirma Rawls, existem muitos aspectos de nossa natureza que poderiam ser considerados como significativos para caracterizar a natureza humana: o homo politicus, o homo economicus, o homo faber. Não recorrendo a nenhuma dessas definições essencialistas ou antropoçógicas de pessoa, o que justifica o conceito de pessoa adotado por Rawls é o próprio propósito da justiça como equidade: "the aim is to work out a conception of political and social justice which is congenial to the most deep-seated convictions and traditions of a modern democratic state. The point of doing this is to see whether we can resolve the impasse in our recent political history; namely, that there is no agreement on the way basic social institutions should be arranged if they are to conform to the freedom and equality of citizens as persons" (RAWLS, 1993, p. 300).

Tendo esse propósito, o conceito de pessoa é justificado de duas formas: como uma idéia intuitiva presente na cultura política pública de uma democracia constitucional e como uma idéia da razão prática. ${ }^{5} \mathrm{O}$ conceito de pessoa é considerado como parte de uma concepção de justiça política e social, diz como os cidadãos devem ver a si mesmos e uns aos outros em suas relações políticas e sociais, da maneira especificada pela estrutura básica da sociedade. Este é o objeto primeiro da justiça, e abrange, com vimos, as principais instituições sociais - a família, o regime econômico, a constituição e a ordem legal, a especificação da propriedade, o sistema político e congêneres - e como essas instituições se combinam para formar um sistema eqüitativo de cooperação social. O que é próprio da estrutura básica é que ela oferece o quadro para um sistema autossuficiente de cooperação para todos os objetivos essenciais da vida humana, objetivos esses realizados pelo grande número de associações e grupos no interior desse quadro.

O que me parece importante aqui é levar em conta que, ao ter como objeto a estrutura básica da sociedade, a justiça como equidade visa levar a sério os padrões de comportamento e a posição real das pessoas no mundo, fortemente influenciados pelo sistema de instituições sociais, econômicas e políticas produtor e reprodutor das vulnerabilidades que afetam a capacidade das pessoas serem sujeitos autônomos, livres e iguais. Ou seja, o objeto da teoria rawlsiana da justiça não são as pessoas como indivíduos racionais abstratos orientados pelas suas preferências, mas a estrutura básica 
da sociedade - os contextos de individuação e socialização das pessoas - que deve ter como finalidade tornar as pessoas capazes de serem membros normais e plenamente cooperativos da sociedade. A capacidade para a cooperação social é vista como fundamental, uma vez que se adote a estrutura básica como objeto primeiro da justiça. Para as pessoas serem vistas dessa maneira, a teoria da justiça deve considerar, como vimos, que as pessoas estão dotadas de duas capacidades morais - o senso de justiça (a disposição para seguir princípios de justiça e de dar e receber razões para justificá-los) e a concepção do bem (de formar, rever e perseguir certo plano racional de vida), que constituem a base da cidadania igual. O que se espera é que a estrutura básica esteja organizada de forma justa de modo a eliminar as arbitrariedades morais decorrentes de relações de dominação social e política que impedem as pessoas de participar efetivamente da cooperação social voltada para a vantagem mútua, mas com base no respeito mútuo, com a disposição de respeitar os termos equitativos e apropriados de cooperação.

Diante disso, "the problem of specifying the basic liberties and grounding they priority can be seen as the problem of determining apropriate fair terms of cooperation on the basis of mutual respect" (RAWLS, 1993, p. 303). Até as guerras religiosas dos séculos XVI e XVII, esses termos equitativos eram muito restritos: a cooperação social com base no respeito mútuo era considerada impossível entre aqueles que professavam uma fé diferente ou uma concepção do bem diferente. $\mathrm{O}$ liberalismo nasce neste contexto com o desenvolvimento de diversos argumentos a favor da tolerância religiosa. No século XIX, com Constant, Tocqueville e Mill, a moralidade política liberal foi aprimorada tendo em vista o contexto do Estado democrático moderno em nascimento. Um dos pressupostos centrais do liberalismo "is that equal citizens have different and indeed incommensurable and irreconcilable conceptions of the good. In a modern democratic society the existence of such diverse ways of life is seen as a normal condition which can only be removed by the autocratic use of state power" (RAWLS, 1993, p. 303).

A moralidade política liberal aceita esse pluralismo de concepções do bem como um fato da vida moderna; considera-o como algo desejável desde que a pluralidade possa ser acomodada num sistema de liberdades fundamentais iguais, de modo que sejam realizados os muitos benefícios da diversidade humana. Uma teoria da justiça deve 
fornecer princípios justificados de modo razoável e público a fim de que "the social union is no longer founded on a conception of the good as given by a common religious faith or philosophical doctrine, but on a shared public conception of justice appropriate to the conception of citizens in a democratic state as free and equal persons" (RAWLS, 1993, p. 304). O ideal político a ser realizado no mundo social é o da autonomia plena dos cidadãos: "full autonomy include not only this capacity to be rational but also the capacity to advance our conception of the good in ways consistent with honoring the fair terms of social cooperation; that is, the principles of justice" (RAWLS, 1993, p. 306).

Como sabemos, Rawls elabora um procedimento complexo de justificação que abarca diferentes níveis de generalidade para justificar a escolha dos princípios de justiça: o procedimento de deliberação e escolha dos princípios na posição original e o método do equilíbrio reflexivo cujo ápice é o consenso sobreposto. Mas mais do que um procedimento, Rawls introduz elementos substantivos na justificação dos princípios: os bens primários (ou bens básicos). "The main idea is that primary goods are singled out by asking which things are generally necessary conditions and all-purpose means to enable persons to pursue their determinate conceptions of the good and to develop and exercise their two moral powers" (RAWLS, 1993, p. 307) Os bens primários são: a) as liberdades fundamentais, como condições institucionais essenciais e necessárias para o desenvolvimento e exercício pleno e bem informado das duas capacidades morais dos cidadãos; são indispensáveis para a proteção de um amplo leque de concepções específicas do bem; b) a liberdade de movimento e de escolha de ocupação num contexto de oportunidades variadas, que permitem a realização de diversos fins últimos e a possibilidade de levar a cabo uma decisão de revisá-los e mudá-los, se o desejarmos; c) os poderes e prerrogativas de posições e cargos de responsabilidade, que abrem espaço para várias capacidades sociais e de autonomia do self; d) renda e riqueza, entendidos como meios polivalentes necessários para realizar direta ou indiretamente uma grande variedade de fins, quaisquer que sejam; e) as bases sociais do auto-respeito, que incluem aqueles aspectos das instituições básicas em geral essenciais para que os cidadãos tenham um vigoroso sentimento de seu próprio valor como pessoas, e para que sejam capazes de desenvolver e exercer suas capacidades morais e de promover seus objetivos e fins com auto-confiança. 
O argumento de Rawls (1993, p. 309) é que "the basic liberties are indeed primary goods", e sua importância é justificada enquanto condições formais e materiais necessárias ao desenvolvimento da autonomia plena das pessoas como cidadãos. Em outros termos, as liberdades fundamentais e sua prioridade e o valor equitativo das liberdades políticas são justificadas tendo em vista sua importância no desenvolvimento das duas capacidades morais que caracterizam os cidadãos livres e iguais de uma sociedade democrática e assim são fundamentais para estabilizar uma cooperação social justa.

O que eu gostaria de destacar nessa argumentação é que Rawls não está pensando apenas na questão redistributiva, mas principalmente nas bases sociais do autorrespeito. As liberdades fundamentais representam o conjunto de condições materiais e institucionais para a realização da autonomia, mas mais do que isso: elas desempenham um papel importante na promoção do autorrespeito, uma dimensão de auto-relação prática consigo mesmo que é fundamental para explicar como os indivíduos adquirem também um sentido interno de sua autonomia. ${ }^{6}$ Rawls define o autorrespeito a partir de dois aspectos: "First ... it includes a person's sense of his own value, his secure conviction that his conception of his good, his plan of life is worth carrying out. And second, self-respect implies confidence in one's ability, so far as it is within one's power, to fullfill one's intentions" (RAWLS, 2003, p. 386). Ele se baseia em nossa autoconfiança enquanto membros plenamente cooperativos da sociedade, proporcionando um sentimento seguro do próprio valor, uma convicção firme de que vale a pena procurar realizar sua própria concepção do bem. Ele depende de e é incentivado por certas características públicas das instituições sociais básicas, de como elas funcionam juntas e como se espera que as pessoas aceitem esses arranjos, considerem e tratem umas às outras. Isso porque o sentimento de nosso próprio valor, assim como nossa autoconfiança, depende do respeito e da reciprocidade que os outros demonstram ter por nós, pois "unless we feel that our endeavors are respected by them, it is difficult if not impossible for us to maintain the conviction that ours ends are worth advancing" (RAWLS, 2003, p. 155-56).

Rawls esclarece que já no nível mais fundamental os indivíduos exigem a aprovação dos outros: o autorrespeito é construído primeiramente na tenra infância, 
quando os pais afirmam o valor de seus filhos ao expressarem o desejo de cuidar e encorajar suas atividades (RAWLS, 2003, p. 406). No contexto mais amplo das instituições sociais, medimos o reconhecimento dos outros por nossa personalidade tomando como referência o status ou nosso lugar na cooperação social. Como é impossível assegurar o autorrespeito dos indivíduos pela igualdade de status em todos os níveis possíveis, Rawls argumenta, num primeiro nível, que a distribuição igual de direitos e liberdades fundamentais fornece uma igualdade de status que satisfaz a necessidade de autorrespeito nos âmbitos mais fundamentais: "the basis of self-respect in a just society is not then one's income share but the publicity affirmed distribution of fundamental rights and liberties. And this distribution being equal, everyone has a similar and secure status when they meet to conduct common affairs of the wider society. No one is inclined to look beyond the constitutional affirmation of equality further political ways of securing his status. Nor, on the other and, are men disposed to acknowledge a less than equal liberty" (RAWLS, 2003, p. 477). A aposta de Rawls é que as liberdades iguais possibilitam a criação de um ambiente associativo rico e diversificado no qual os indivíduos podem cultivar e adquirir confiança em suas capacidades e habilidades, uma parte necessária para terem um senso seguro de seu próprio valor. Em suma, as liberdades iguais fundamentais que asseguram as condições sociais e políticas que tornam possível que os cidadãos expressem seu respeito mútuo uns pelos outros enquanto indivíduos razoáveis e dignos de confiança, assim como seu reconhecimento do valor que todos os cidadãos atribuem à própria forma de vida. Elas servem ao propósito público comum de assegurar a justiça a cada cidadão, enquanto pessoa livre e igual, em uma base de respeito mútuo, fazendo com que os vínculos de reciprocidade se estendam à sociedade como um todo. Em suma, levando em conta essa compreensão das liberdades básicas, fica mais ou menos evidente que Rawls não está pensando apenas nas dimensões externas (materiais e institucionais) da autonomia, mas também em como as pessoas, na qualidade de membros livres e iguais de um sistema de cooperação social, podem também desenvolver um senso interno de autonomia quando as bases sociais do autorrespeito estiverem asseguradas na estrutura básica da sociedade, isto é, quando os contextos de socialização e as relações intersubjetivas de reconhecimento e de respeito entre as pessoas estiverem asseguradas e livres de relações de dominação social e políticas arbitrárias.

ethic@ - Florianópolis v.13,n.1, p. 74 -90, Jun. 2014. 
Mas, é claro, as liberdades fundamentais não são meras formalidades. A concepção da justiça como equidade preocupa-se, principalmente no segundo princípio, com os meios materiais necessários para os cidadãos usarem efetivamente suas liberdades iguais e realizarem seus planos de vida. Muitos argumentaram, principalmente os democratas radicais e os socialistas, que, embora possa parecer que os cidadãos são efetivamente iguais, são demasiado grandes as desigualdades sociais e econômicas e que aqueles com maior riqueza e renda podem controlar a elaboração legislativa em benefício próprio.

É justamente por ter em vista esse tipo de assimetria no uso efetivo das liberdades que Rawls faz a distinção entre liberdades fundamentais e o valor das liberdades. As liberdades fundamentais especificam os direitos e deveres institucionais entre os cidadãos. Obviamente a falta de informação, a pobreza e a ausência de meios materiais em geral impedem as pessoas de exercerem seus direitos e de tirar proveito dessas possibilidades. São fatores que atingem o valor das liberdades, isto é, o proveito que as pessoas tiram de suas liberdades. Embora as liberdades fundamentais sejam as mesmas para todos os cidadãos, o valor da liberdade não é o mesmo para todos. Para tornar mais efetivo o uso das liberdades, Rawls confere atenção especial à dimensão da autodeterminação política. Apenas as liberdades políticas merecem um tratamento especial por parte de Rawls, "by including in the first principle of justice the guarantee that the political liberties, and only these liberties are secured by what I have called their "fair value"” (RAWLS, 1993, p. 327), no sentido de que todos os cidadãos tenham uma oportunidade equitativa de assumir um cargo público e influenciar o resultado de decisões políticas.

A garantia do valor equitativo das liberdades políticas tem como principal finalidade assegurar "for each citizen a fair and roughly equal Access to the use of a public facility designed to serve a definite political purpose, namely, the public facility specified by the constitutional rules and procedures which govern the political process and control the entry to positions of political authority" (RAWLS, 1993, p. 328). Uma vez que consideramos o papel peculiar do processo político na determinação das leis e políticas que devem regular a estrutura básica, torna-se mais evidente para Rawls que as liberdades políticas devem ser objeto da garantia especial do valor equitativo. Claro, Rawls não está afirmando que a vida política e a participação de todos no autogoverno 
sejam consideradas bens proeminentes para cidadãos plenamente autônomos. A valorização da participação política em si é uma concepção do bem entre outras. O que importa a Rawls é a idéia de um sistema de liberdades básicas iguais. "The garantee of fair value for the political liberties is included in the first principle of justice because it is essential in order to establish just legislation and also to make sure that the fair political process specified by the constitution is open to everyone on a basis of rough equality" (RAWLS, 1993, p. 330).

As liberdades fundamentais especificam o status comum e garantido dos cidadãos iguais numa sociedade democrática bem ordenada. "The liberty is a certain pattern of social forms" (RAWLS, 2003, p. 86). Ela é produzida pelas instituições sociais, políticas e jurídicas da sociedade. A ênfase está na organização de uma sociedade justa e livre. Segundo uma sugestão de Albrecht WELLMER (1996), a compreensão que Rawls tem da realização das liberdades é muito próxima ao modo de Hegel entender a realização da ideia de liberdade na Filosofia do Direito. Uma vez que o objeto primeiro da justiça é a estrutura básica da sociedade - que abrange, como vimos antes, as instituições da família, da propriedade, do mercado e do rule of law - podemos ver no liberalismo político de Rawls uma concepção de justiça que abrange os vários momentos ou dimensões de realização de uma concepção universal de liberdade comum, que acabaria se concretizando numa eticidade democrática. Ela abrange diferentes esferas de autorrealização dos seres humanos. Mas diferente de Hegel, em Rawls a passagem do direito abstrato à eticidade concreta se daria de forma mais democrática, já que o princípio da liberdade igual conduz diretamente a um princípio de direitos iguais de participação política e de deliberação pública.

Nesse sentido, a questão da justiça não parece se resumir apenas à criação de condições de autorrealização ética dos indivíduos que asseguram a satisfação de suas necessidades e carências básicas. O objeto de uma teoria da justiça é antes a estrutura básica profunda da sociedade que produz e reproduz as carências e as vulnerabilidades das pessoas. Por conseguinte, a tarefa de uma teoria da justiça é fazer com que a própria estrutura básica da sociedade assegure às pessoas a possibilidade real de definirem social e politicamente sua vida em comum. Portanto, a questão central da justiça é também a questão do poder social e político. Não se trata de saber apenas quais "bens" devem ser 
legitimamente distribuídos para quem e sob quais razões, mas também de ver como os bens são produzidos socialmente e quem e como são definidas as regras de sua distribuição (FORST, 2011). Ao assumir a estrutura básica da sociedade -as principais instituições sociais, econômicas e políticas - como objeto da justiça, Rawls coloca no centro de sua teoria a questão política acerca da definição da estrutura de produção e de distribuição dos bens e da justificação pública do poder nas sociedades democráticas.

$\mathrm{O}$ enfoque de uma teoria crítica da justiça que evite tanto o caráter excessivamente abstrato alheio ao contexto quanto o particularismo de uma concepção de vida boa obcecada pelo contexto precisa considerar as necessidades e demandas que podem surgir dos contextos de socialização dos indivíduos e que podem ser justificadas publicamente segundo razões aceitáveis recíproca e universalmente em diferentes contextos normativos. Esta é muito mais uma tarefa prática do que uma tarefa teórica, que implica um momento irredutível da autonomia em todas as relações de justificação. Poder-se-ia dizer que ser uma pessoa que tem o direito à justificação é outro elemento constitutivo das bases sociais do autorrespeito. A questão central de uma teoria crítica da justiça não é promover a autorrealização ética ou a autonomia individual negativa dos indivíduos, mas sim fazer com que a estrutura básica da sociedade não determine arbitrariamente a vida das pessoas. Parece-nos ser esta também a preocupação que está na base do liberalismo político de Rawls: a justiça não tem a ver com a promoção da vida boa, mas com a primazia das liberdades básicas na organização da estrutura básica da sociedade que salvaguarda as pessoas das vulnerabilidades internas e externas relacionadas à autonomia, ao proporcionar, entre outras coisas, as bases sociais do autorrespeito. 


\begin{abstract}
Notas:
${ }^{1}$ Professor da Universidade Federal de Santa Catarina, Florianópolis, Santa Catarina, Brasil. Email:dlwerle@yahoo.com.br
\end{abstract}

${ }^{2}$ HONNETH e ANDERSON (2005, p.142) pretendem justamente aprimorar a abordagem básica de Rawls, e não se afastar dela. Sugerem três revisões no modelo de Rawls para acomodar a concepção de autonomia baseada no reconhecimento: 1) ele precisa ser mais aberto a considerações baseadas naquilo que sabemos sobre as pessoas humanas; 2) ele precisa tratar mais extensamente dos modos pelos quais a infraestrutura do reconhecimento da sociedade pode deixar a autonomia dos indivíduos inaceitavelmente vulnerável; e 3) é preciso admitir que a ênfase nas relações sociais de reconhecimento exige um afastamento de questões exclusivamente distributivas. Para ver se Honneth e Anderson realmente conseguem se afastar da concepção de Rawls, cf. o excelente artigo de BANKOVSKY (2011).

${ }^{3}$ É importante ter em mente que o autorrespeito não desempenha somente esse papel na teoria da justiça de Rawls. Para além da justificação das liberdades básicas, Rawls recorre à noção de auto-respeito para reforçar o argumento da estabilidade. Rawsl "argues that justice as fairness is a stable conception of justice because it cultivates and supports individual's self-respect in the most reliable way. As a result, individuals in a society organized by his principles of justice will be psychologically disposed and motivated to uphold those principles and institutional arrangements that have so effectively underwritten their sense of selfworth" (ZINK, 2011, p. 331-332).

${ }^{4}$ Essa indeterminação e o conflito entre as liberdades são resolvidos não pela teoria, mas pelo poder político, exercido segundo um princípio liberal de legitimidade baseado na razão pública (WERLE, 2011).

${ }^{5}$ The political constructivism "says that once, if ever, reflective equilibrium is attained, the principles of political justice (content) may be represented as the outcome of a certain procedure of construction (structure). In this procedure, as modeled by the original position, rational agents, as representatives of citizens and subject to reasonable conditions, select the public principles of justice to regulate the basic strucutre of society. This procedure, we conjecture, embodies all the relevant requirements of practical reason an shows how the principles of justice follow from the principles of practical reason in union with conceptions of society and person, themselves ideas of practical reason" (RAWLS, 1993, p. 89-90). Noutra passagem do mesmo livro, Rawls reforça essa ideia: "Let us say, then, that the conceptions of society and person, and the public role of principles of justice, are ideas of practical reason. Not only do they assume a form that practical reason requires for its application, but they provide the context within which practical questions and problems arise. (...) Without the ideas of society and person, conceptions of the right and the good have no place. They are as basic as the ideas of judgment and inference, and the principles of practical reason" (RAWLS, 1993, p. 110) Essas passagens indicam que a ancoragem histórica da dimensão kantiana não redunda numa concepção conservadora e contextualista da justiça, ou numa espécie de concepção realista da moral. Rawls se opõe expressamente ao realismo moral. O construtivismo kantiano sustenta que a objetividade moral deve ser compreendida segundo um ponto de vista social construído adequadamente, que todos poderiam aceitar. Fora do procedimento de construção dos princípios de justiça, não existem fatos morais. Como dito acima, esse procedimento é construído segundo ideias de sociedade e de pessoa que são simultaneamente ideias da razão prática - e, como tais, pressupostos indispensáveis na formação do juízo moral imparcial - e ideias intuitivas implícitas na cultura política das sociedades democráticas modernas. Portanto, Rawls parece ter em mente um procedimento complexo de justificação que procurar reunir, na base comum da ideia fundamental de sociedade como sistema de cooperação social equitativo e de pessoa moral livre e igual, uma interdependência "dialética" entre o ponto de vista moral do que é bom para todos e o ponto de vista do que é bom para nós. Trata-se de uma tentativa de mostrar que os princípios de justiça podem ser justificados "em todos os níveis de generalidade". Cf. WERLE (2008).

ethic@ - Florianópolis v.13,n.1, p. 74 -90,Jun. 2014. 
${ }^{6}$ Uma série de intérpretes tem acentuado o papel justificativo do auto-respeito na teoria da justiça de Rawls. Cf. COHEN, 1989; KEAT e MILLER, 1974; MASSEY, 1983; MILLER, 1978; NIELSON, 1979; ZAINO, 1998; EYAL, 2005; SHUE, 1975.

ethic@ - Florianópolis v.13, n.1, p. 74 -90, Jun. 2014. 


\section{Referências bibliográficas:}

BANKOVSKY, M. (2011). "Social justice: defending Rawls' theory of justice against Honneth's objections". In: Philosophy \& Social Criticism, v. 37, n. 1, pp. 95-118.

COHEN, J. (1989). “Democratic equality”. In: Ethics, v. 99, n. 4, pp. 727-51.

HART, H. L. A. (1975). "Rawls on liberty and its priority" in: Daniels, Norman (ed.) Reading Rawls. Oxford: Basil Blackwell.

EYAL, N. (2005). “ 'Perhaps the most important primary good': self-respect and Rawls's principles of justice”. In: Politics, Philosophy \& Economics, v. 4, n. 2, pp. 195-219.

FORST, R. (2011). Kritik der Rechtfertigungsverhältnisse - Perspektiven einer kritischen Theorie der Politik. Frankfurt am Main: Suhrkamp.

HONNETH, A.; ANDERSON, J. (2005). "Autonomy, vulnerability, recognition and justice". In: CHRISTMAN, J.; ANDERSON, J. Autonomy and the challenges to liberalism: new essays. Cambridge: Cambridge University Press, pp. 127-49.

KEAT, R.; MILLER, D. (1974). “ Understading justice”. In: Political Theory, v. 2, n.1, pp. 3-31.

MASSEY, S. (1983). "Is self-respect a moral or a psychological concept?". In: Ethics, v. 93, n. 2, pp. 246-61.

MILLER, D. (1978). "Democracy and social justice". In: British Journal of Political Science, v.8, n.1, pp. 1-19.

NIELSON, K. (1979). "Radical egalitarian justice: justice as equality". In: Social Theory and Practice, v. 5, n.2, pp. 209-26.

RAWLS, J. (1993). Political Liberalism. New York: Columbia University Press. . (2003) A theory of justice. (Sixth printing).Cambridge/Mass.: The Bleknap Press of Harvard University Press.

SHUE, H. (1975). “Liberty and self-respect”. In: Ethics, v. 85, n.3, pp. 195-203.

WELLMER, A. (1996) "Condiciones de una cultura democrática: sobre el debate entre 'liberales' y 'comunitaristas"'. In:WELLMER, A. Finales de Partida: La Modernidad Irreconciliable. Tradução: Manuel Jímenez Redondo. Madrid: Cátedra, p.77-101.

WERLE, D. L. (2008). Justiça e democracia. Ensaios sobre John Rawls e Jürgen Habermas. São Paulo: Esfera Publica. 
. (2011). "Liberdades básicas, justificação pública e poder político em John Rawls". In: Dissertatio, v. 34, pp. 183-207.

ZAINO, J. (1998). "Self-respect and rawlsian justice". In: Journal of Politics, v. 60, n. 3, pp. 737-53.

ZINK, J. R. (2011). "Reconsidering the role of self-respect in Rawls's A theory of Justice". In: The Journal of Politics, v. 73, n.2, pp. 331-344.

Recebido / Received: 12/05/2014

Aprovado / Approved: 12/06/2014

ethic@ - Florianópolis v.13, n.1, p. 74 -90, Jun. 2014. 